\title{
Statefinder Diagnostic for Dark Energy Models in Bianchi I Universe
}

\author{
M. Sharif *and Rabia Saleem ${ }^{\dagger}$ \\ Department of Mathematics, University of the Punjab, \\ Quaid-e-Azam Campus, Lahore-54590, Pakistan.
}

\begin{abstract}
In this paper, we investigate the statefinder, the deceleration and equation of state parameters when universe is composed of generalized holographic dark energy or generalized Ricci dark energy for Bianchi I universe model. These parameters are found for both interacting as well as non-interacting scenarios of generalized holographic or generalized Ricci dark energy with dark matter and generalized Chaplygin gas. We explore these parameters graphically for different situations. It is concluded that these models represent accelerated expansion of the universe.
\end{abstract}

Keywords: Dark energy models; Statefinder parameters.

PACS: 95.36.+x; 95.35.+d.

\section{Introduction}

Astronomical observations of Type Ia supernova [1] indicate that our universe is expanding with accelerating velocity rather than slowing down. This cosmic expansion is confirmed by some other independent observations like CMBR [2], SDSS [3, WMAP [4] etc. An exotic form of matter with large negative pressure is found to be responsible for this cosmic expansion known

*msharif.math@pu.edu.pk

†rabiasaleem1988@yahoo.com 
as dark energy (DE) which occupies $2 / 3$ of the total energy of our universe. All attempts indicate that nature as well as origin of DE is still enigmatic and is a mystery for scientists. The simplest candidate for DE is the cosmological constant with equation of state $(\mathrm{EoS}) \omega=-1$. The value of this cosmological constant is very small as compared to quantum field theory which is known as cosmological constant problem, i.e., fine-tuning and cosmic-coincidence problems. The nature of DE has been explored by classifying the behavior of EoS parameter like quintessence [5], k-essence [6], tachyon field [7], phantom model [8] and Chaplygin gas [9] etc. However, none of these models is very successful.

Holographic principle [10] is a good attempt in this direction which explains some problems of cosmological constant and DE. According to this principle, the degree of freedom in a bounded system should be finite and it scales with its boundary area but not with its volume. Cohen et al. [11] proposed a relationship between ultraviolet (UV) and infrared (IR) cut-offs due to a limit set by the formation of a black hole, i.e., $L^{3} \rho_{\nu} \leq L M_{p}^{2}$. Here $\rho_{\nu}$ is the vacuum energy density associated with UV cut-off, $L$ is the IR cut-off and $M_{P}=(8 \pi G)^{-\frac{1}{2}}$ is the reduced Planck mass.

In order to discuss an accelerated universe, three different values of $L$ have been introduced, i.e., apparent, particle and event horizon. It is found [12 that the first two horizons do not give accelerated expansion of the universe. The best choice is the future event horizon for which $\omega<-\frac{1}{3}$, a sufficient condition for cosmic acceleration. Zhang [13] pointed out that our universe is bounded by the future event horizon for which vacuum energy density is converted to holographic DE (HDE) density, i.e., $\rho_{\nu}=3 c^{2} M_{p}^{2} L^{-} 2$, where $c$ is a dimensionless parameter. This model has been used to investigate the cosmic evolution by different people [14].

Holographic DE looks reasonable as it resolves some problems related to DE but it also suffers with causality problem, i.e., future event horizon is presumed in this model. Gao et al. [15] gave the idea that DE density and Ricci scalar are proportional to each other, i.e., $\rho_{x} \propto R$ - known as Ricci dark energy (RDE). This model of DE is phenomenological viable as it gives results consistent with observational data. It also alleviates the causality as well as cosmic coincidence problem. The Ricci scalar for flat universe is $6\left(\dot{H}+2 H^{2}\right)$ for which RDE density becomes $\rho_{r}=3 c^{2}\left(\dot{H}+2 H^{2}\right)$. Chattopadhyay [16] showed that when the generalized RDE (GRDE) is considered in HoravaLifshitz gravity, it behaves like quintessence for $c^{2}>,=,<\frac{1}{2}$.

Feng and Li [17] investigated viscous RDE model by assuming that there 
is a bulk viscosity in the linear barotropic fluid and RDE. The RDE model can be obtained by choosing the causal scale as IR cut-off. Kim et al. [19] showed that the accelerating phase of the RDE is that of a constant DE model for FRW universe. Recent work in RDE and HDE models is demonstrated in [20]. $\mathrm{Xu}$ et al. [21] proposed two models of DE, i.e., generalized HDE (GHDE) and GRDE whose energy densities are

$$
\rho_{h}=3 c^{2} m_{p}^{2} H^{2} f\left(\frac{R}{H^{2}}\right), \quad \rho_{r}=3 c^{2} m_{p}^{2} R g\left(\frac{H^{2}}{R}\right),
$$

where $\mathrm{c}$ is a constant, $f(x)=\alpha x+(1-\alpha), g(y)=\beta y+(1-\beta),(\alpha, \beta$ are constants) are positive functions. We can also recover the original energy densities of GHDE and GRDE by assuming $\alpha, \beta=0,1$. Notice that GHDE model is converted into GRDE model by replacing $\alpha$ with $1-\beta$. In a recent paper [22], the accelerating universe is investigated through the deceleration and statefinder parameters for GHDE and GRDE models by using FRW metric.

In this paper, we consider LRS Bianchi I (BI) universe model composed of DM, generalized Chaplygin gas (GCG) and DE with GHDE/GRDE model to discuss evolution of the universe. The plan of the paper is as follows: In section $\mathbf{2}$, the statefinder parameters are calculated for the combination of two fluids. Section 3 is devoted to explore the EoS, the deceleration parameters and statefinder diagnostic pair for GHDE/GRDE model without DM. We investigate these parameters with DM in non-interacting and interacting scenarios in section $\mathbf{4}$. In section $\mathbf{5}$, these parameters are discussed when the universe is composed of GHDE/GRDE with GCG in non-interacting and interacting scenarios. In the last section, we summarize the results.

\section{Statefinders for Two Fluid System}

Our universe appears homogeneous and isotropic on large scale at the present time. The existence of the anisotropy at early times is a natural phenomenon. We observe the anisotropy in galaxies, clusters and super clusters today. It would be appropriate to discuss a geometry that is more general than the isotropic and homogeneous FRW geometry. A Bianchi type I model being the straightforward generalization of the flat FRW model, is one of the simplest models of the anisotropic universe. This model describes a homogeneous, spatially flat and anisotropic universe. 
In this section, we formulate the field equations, deceleration parameter and statefinder parameters for LRS BI universe model. We assume that the fluid is a combination of DM and DE. The line element of BI model is given as follows

$$
d s^{2}=-d t^{2}+A^{2}(t) d x^{2}+B^{2}(t)\left(d y^{2}+d z^{2}\right),
$$

where $A$ and $B$ are scale factors. We use the well-known condition $A=B^{m}$ [23], where $m \neq 1$ is a positive constant. Consequently, the above metric reduces to

$$
d s^{2}=-d t^{2}+B^{2 m}(t) d x^{2}+B^{2}(t)\left(d y^{2}+d z^{2}\right) .
$$

The field equations corresponding to perfect fluid turn out to be

$$
\begin{array}{r}
(2 m+1) \frac{\dot{B}^{2}}{B^{2}}=8 \pi \rho, \\
2 \frac{\ddot{B}}{B}+\frac{\dot{B}^{2}}{B^{2}}=-8 \pi p, \\
m^{2} \frac{\dot{B}^{2}}{B^{2}}+(m+1) \frac{\ddot{B}}{B}=-8 \pi p .
\end{array}
$$

Equation (2) can be written as

$$
H_{2}^{2}=\frac{1}{1+2 m}\left(\rho_{m}+\rho_{X}\right), \quad H_{2}=\frac{\dot{B}}{B}
$$

where $\mathrm{H}_{2}$ is the directional Hubble parameter while $\rho_{m}$ and $p_{m}$ are the energy density and pressure of matter respectively, $\rho_{X}=\rho_{h}, p_{X}=p_{h}$ are the energy density and pressure for GHDE and $\rho_{X}=\rho_{r}, p_{X}=p_{r}$ are the energy density and pressure for GRDE, respectively. The conservation equation yields

$$
\dot{\rho}_{m}+\dot{\rho}_{X}+(m+2)\left(\rho_{m}+\rho_{X}+p_{m}+p_{X}\right) H_{2}=0 .
$$

Taking derivative of Eq.(5) and using (6)

$$
\dot{H}_{2}=-\frac{(m+2)}{2(1+2 m)}\left(\rho_{m}+\rho_{X}+p_{m}+p_{X}\right) \text {. }
$$

Sahni et al. 24] introduced a new dimensionless static, statefinder, which can differentiate between different types of DE models and might be a good diagnostic of cosmological models. As the statefinder diagnostic pair depends upon the scale factor, so we can say that this pair is a geometrical diagnostic 
in the sense that it is constructed from a spacetime metric directly. This pair examines expansion of the universe at large scale as it involves third derivative of the scale factor $B(t)$.

The deceleration parameter, $q$, and statefinder diagnostic pair $\{r, s\}$ in terms of scale factor are formulated as follows

$$
\begin{aligned}
q & =-\left[\frac{(m-1)}{(m+2)}+\frac{3 B \ddot{B}}{(m+2) \dot{B}^{2}}\right] \\
r & =\frac{(m-1)(m-4)}{(m+2)^{2}}+\frac{9(m-1) B \ddot{B}}{(m+2)^{2} \dot{B}^{2}}+\frac{(m+2) B^{2} \dddot{B}}{3 \dot{B}^{3}} \\
s & =\frac{r-1}{3\left(q-\frac{1}{2}\right)} .
\end{aligned}
$$

These can be expressed in the form of pressure and density as

$$
\begin{aligned}
q & =\frac{1}{2}+\frac{3}{2}\left(\frac{p_{m}+p_{X}}{\rho_{m}+\rho_{X}}\right), \\
r & =\left[\frac{3 m^{5}+21 m^{4}+54 m^{3}-3 m^{2}+15 m+72}{18(m+2)^{2}}\right] \\
& +\left[\frac{-9(m-1)}{2(m+2)}+\frac{(m-1)(m+2)^{2}}{9}+\frac{(m+2)^{3}}{18}-\frac{(m+2)}{2}\right]\left(\frac{p_{m}+p_{X}}{\rho_{m}+\rho_{X}}\right) \\
& +\frac{(m+2)^{3}}{6\left(\rho_{m}+\rho_{X}\right)}\left[\frac{\partial p_{m}}{\partial \rho_{m}}\left(p_{m}+\rho_{m}\right)+\frac{\partial p_{X}}{\partial \rho_{X}}\left(p_{X}+\rho_{X}\right)\right], \\
s & =\left[\frac{-(m-1)}{(m+2)}+\frac{2(m-1)(m+2)^{2}}{81}+\frac{(m+2)^{3}}{81}-\frac{(m+2)}{9}\right] \\
& +\left[\frac{3 m^{5}+21 m^{4}+54 m^{3}-21 m^{2}-57 m}{81(m+2)^{2}}\right]\left(\frac{\rho_{m}+\rho_{X}}{p_{m}+p_{X}}\right) \\
& +\frac{(m+2)^{3}}{27\left(p_{m}+p_{X}\right)}\left[\frac{\partial p_{m}}{\partial \rho_{m}}\left(p_{m}+\rho_{m}\right)+\frac{\partial p_{X}}{\partial \rho_{X}}\left(p_{X}+\rho_{X}\right)\right] .
\end{aligned}
$$

\section{GHDE Model without Dark Matter}

Here we evaluate the EoS, the deceleration and statefinder parameters when the universe is filled with GHDE/GRDE only. Equation (5) can be written as

$$
H_{2}^{2}=\frac{1}{1+2 m} \rho_{h}
$$


The Ricci scalar is given by

$$
R=-2\left[\left(m^{2}+2 m+3\right) H_{2}^{2}+(m+2) \dot{H}_{2}\right] .
$$

Using this value of $R$, we get GHDE density as

$$
\rho_{h}=\frac{c^{2}}{3}\left[-18(m+2) \alpha \dot{H}_{2}+\left[(m+2)^{2}-\left(19 m^{2}+40 m+58\right) \alpha\right] H_{2}^{2}\right] .
$$

Inserting this value of $\rho_{h}$ in Eq.(11), we obtain

$$
\dot{H}_{2}+\frac{(1+2 m) H_{2}^{2}}{6 \alpha c^{2}(m+2)}\left[1-\frac{c^{2}}{3(1+2 m)}\left[(m+2)^{2}-\left(19 m^{2}+40 m+58\right) \alpha\right]\right]=0(
$$

whose solution is

$$
H_{2}^{2}=H_{0}^{2} B^{\mu}
$$

where $H_{0}^{2}$ is an integration constant and

$$
\mu=-\frac{1+2 m}{6(m+2) c^{2} \alpha}\left[1-\frac{c^{2}}{3(1+2 m)}\left[(m+2)^{2}-\left(19 m^{2}+40 m+58\right) \alpha\right]\right]
$$

Substituting this value of $H_{2}^{2}$ in Eq.(11), we have

$$
\rho_{h}=(1+2 m) H_{0}^{2} B^{\mu} .
$$

Consequently, Eq.(7) will become

$$
\dot{H}_{2}=-\frac{(m+2)}{2(1+2 m)}\left(\rho_{h}+p_{h}\right)
$$

Inserting the values of $\dot{H}_{2}$ and $\rho_{h}$ from Eqs.(14) and (15), respectively in (16), the pressure of GHDE can be expressed as

$$
p_{h}=-(1+2 m)\left[\frac{\mu}{(m+2)}+1\right] H_{0}^{2} B^{\mu} .
$$

The EoS parameter for GHDE is defined as

$$
\omega_{h}=\frac{p_{h}}{\rho_{h}}=-\left[\frac{\mu}{(m+2)}+1\right]
$$




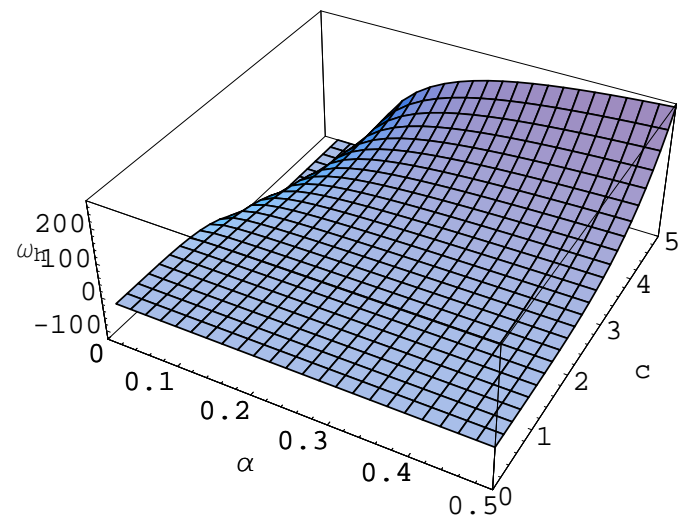

Figure 1: Variation of $\omega_{h}$ against $\alpha$ and $c$.

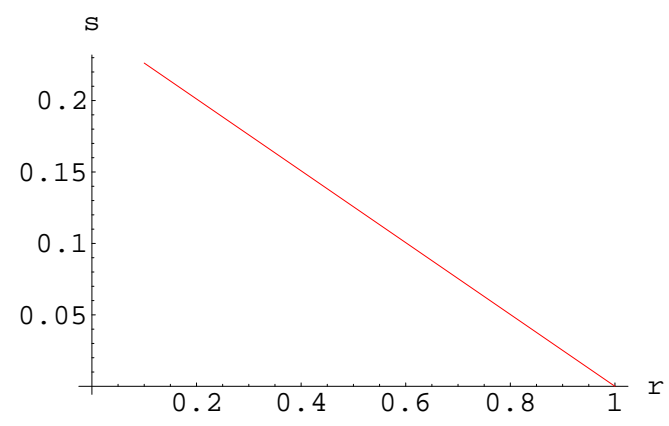

Figure 2: Variation of $r$ against $s$.

which must satisfy $\omega_{h}<-\frac{1}{3}$ to show the expanding universe. Under this condition, we get $\alpha<\frac{(m+2)^{2} c^{2}-3(1+2 m)}{\left(7 m^{2}-8 m+10\right) c^{2}}$ and the model generates DE. The EoS parameter for GHDE is plotted against $\alpha$ and $c$ shown in Figure 1. This shows that $\omega_{h}$ decreases from positive to negative as $\alpha$ decreases and $c$ increases. Equations (15) and (17) lead to

$$
\frac{\partial p_{h}}{\partial \rho_{h}}=\frac{\partial p_{h} / \partial B}{\partial \rho_{h} / \partial B}=\omega_{h}
$$

Using Eqs.(17), (18) and (19) in (8)-(10), we obtain the deceleration param- 


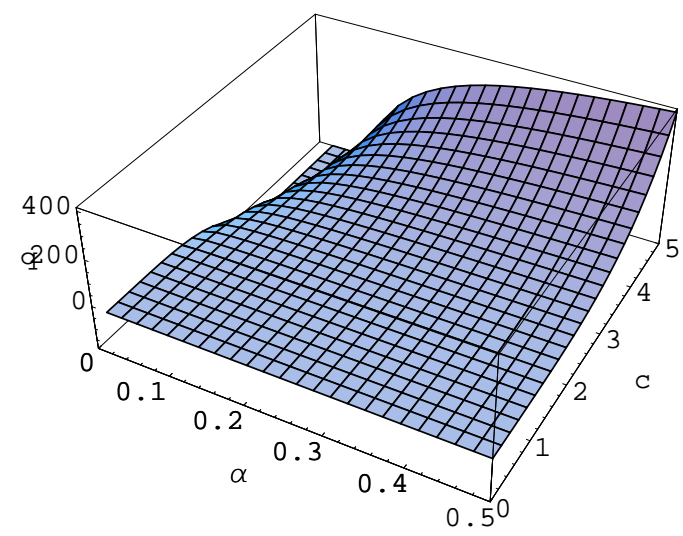

Figure 3: Variation of $q$ against $\alpha$ and $c$.

eter and statefinder diagnostics

$$
\begin{aligned}
q & =-1-\frac{3}{2} \frac{\mu}{(m+2)}, \\
r & =\left[\frac{3 m^{5}+21 m^{4}+54 m^{3}-3 m^{2}+15 m+72}{18(m+2)^{2}}\right] \\
& -\left[\frac{-9(m-1)}{2(m+2)}+\frac{(m-1)(m+2)^{2}}{9}+\frac{(m+2)^{3}}{18}-\frac{(m+2)}{2}\right] \\
& \times\left[\frac{\mu}{(m+2)}+1\right]+\frac{(m+2)^{2} \mu}{6}\left[\frac{\mu}{(m+2)}+1\right], \\
s & =\left[\frac{-(m-1)}{(m+2)}+\frac{2(m-1)(m+2)^{2}}{81}+\frac{(m+2)^{3}}{81}-\frac{(m+2)}{9}\right] \\
& -\left[\frac{3 m^{5}+21 m^{4}+54 m^{3}-21 m^{2}-57 m}{81(m+2)}\right]\left[\frac{1}{\mu+(m+2)}\right] \\
& -\frac{(m+2)^{2} \mu}{27} .
\end{aligned}
$$

The universe will be accelerating if $q<0$, i.e., when $\alpha<\frac{(m+2)^{2} c^{2}-3(1+2 m)}{\left(7 m^{2}-8 m+10\right) c^{2}}$. Figure 2 shows that $s$ decreases as $r$ increases. The deceleration parameter is shown in Figure $\mathbf{3}$ which indicates that $q$ decreases from positive to negative values as $\alpha$ decreases and $c$ increases. If we replace $\alpha$ by $1-\beta$, then all the above solutions are valid for GRDE. In this case, $\beta>\frac{\left(6 m^{2}-12 m+6\right) c^{2}+3(1+2 m)}{\left(7 m^{2}-8 m+10\right) c^{2}}$ for the accelerating universe. 


\section{GHDE Model with Dark Matter}

In this section, we evaluate the above mentioned parameters for non-interacting and interacting scenarios when the universe is a combination of GHDE and DM.

\subsection{Non-Interacting Case}

Here, we assume that the universe is filled with GHDE and non-interacting DM. Consequently, Eq.(5) yields

$$
H_{2}^{2}=\frac{1}{1+2 m}\left(\rho_{h}+\rho_{m}\right)
$$

As there is no interaction between GHDE and DM, so these are independently conserved. Equation (6) leads to conservation equations for DM and GHDE as follows

$$
\dot{\rho}_{m}+(m+2)\left(\rho_{m}+p_{m}\right) H_{2}=0, \quad \dot{\rho}_{h}+(m+2)\left(\rho_{h}+p_{h}\right) H_{2}=0 .
$$

For EoS $p_{m}=\omega_{m} \rho_{m}$, first of the above equation yields

$$
\rho_{m}=\rho_{m_{0}} B^{-(m+2)\left(1+\omega_{m}\right)},
$$

where $\rho_{m_{0}}$ is the constant of integration. Inserting the values of $\rho_{h}$ and $\rho_{m}$ from Eqs.(13) and (22) in Eq.(20), we obtain

$$
\frac{d H_{2}^{2}}{d B}-\frac{\mu}{B} H_{2}^{2}=\frac{\rho_{m_{0}} B^{-(m+2)\left(1+\omega_{m}\right)}}{6 \alpha c^{2}(m+2) B}
$$

whose solution is

$$
H_{2}^{2}=\frac{\rho_{m_{0}} B^{-(m+2)\left(1+\omega_{m}\right)}}{-6 \alpha c^{2}(m+2)^{2}\left[\left[\frac{\mu}{(m+2)}+1\right]+\omega_{m}\right]}+H_{1}^{2} B^{\mu},
$$

where $H_{1}$ is an integrating constant. Combining Eqs.(20), (21)-(23), we obtain density and pressure

$$
\begin{aligned}
& \rho_{h}=\nu \rho_{m_{0}} B^{-(m+2)\left(1+\omega_{m}\right)}+(1+2 m) H_{1}^{2} B^{\mu} \\
& p_{h}=\nu \rho_{m_{0}} \omega_{m} B^{-(m+2)\left(1+\omega_{m}\right)}-(1+2 m)\left[\frac{\mu}{(m+2)}+1\right] H_{1}^{2} B^{\mu}
\end{aligned}
$$




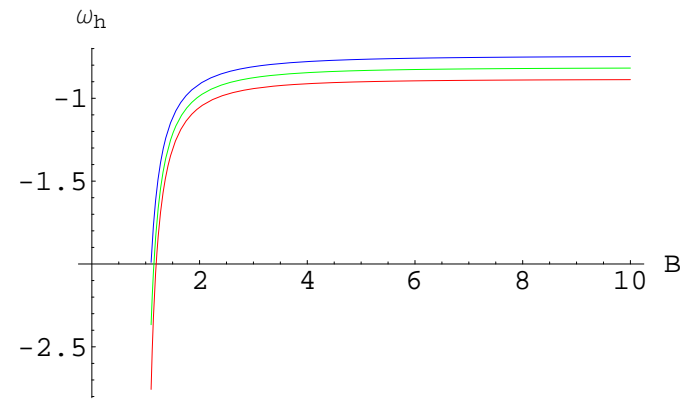

Figure 4: Variation of $\omega_{h}$ against $B$ for $\omega_{m}=0.01, \rho_{m_{0}}=1, H_{1}=1, c=2$, and $\alpha=0.1,0.12,0.15$.

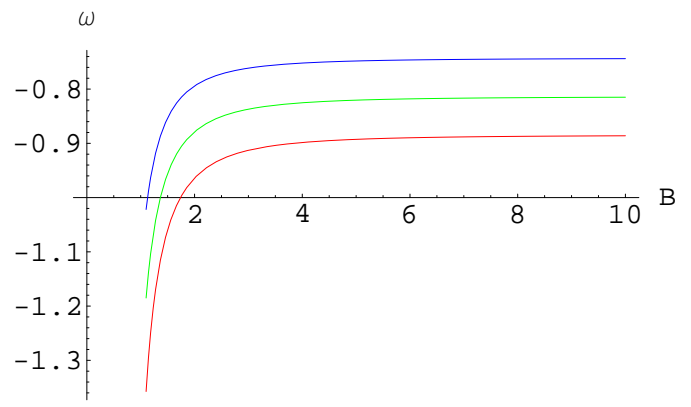

Figure 5: Variation of $\omega$ against $B$ for $\omega_{m}=0.01, \rho_{m_{0}}=1, H_{1}=1, c=2$, and $\alpha=0.1,0.12,0.15$.

where

$$
\nu=\left[\frac{(1+2 m)}{-6 \alpha c^{2}(m+2)^{2}\left[\left[\frac{\mu}{(m+2)}+1\right]+\omega_{m}\right]}-1\right] .
$$

The EoS for GHDE and for the combined fluid is

$$
\begin{aligned}
\omega_{h} & =\frac{p_{h}}{\rho_{h}}=\frac{\nu \rho_{m_{0}} \omega_{m} B^{-(m+2)\left(1+\omega_{m}\right)}-(1+2 m)\left[\frac{\mu}{(m+2)}+1\right] H_{1}^{2} B^{\mu}}{\nu \rho_{m_{0}} B^{-(m+2)\left(1+\omega_{m}\right)}+(1+2 m) H_{1}^{2} B^{\mu}}, \\
\omega & =\frac{p_{h}+p_{m}}{\rho_{h}+\rho_{m}}=\frac{1}{(\nu+1) \rho_{m_{0}} B^{-(m+2)\left(1+\omega_{m}\right)}+(1+2 m) H_{1}^{2} B^{\mu}} \\
& \times(\nu+1) \rho_{m_{0}} \omega_{m} B^{-(m+2)\left(1+\omega_{m}\right)}-(1+2 m) \\
& \times\left[\frac{\mu}{(m+2)}+1\right] H_{1}^{2} B^{\mu} .
\end{aligned}
$$




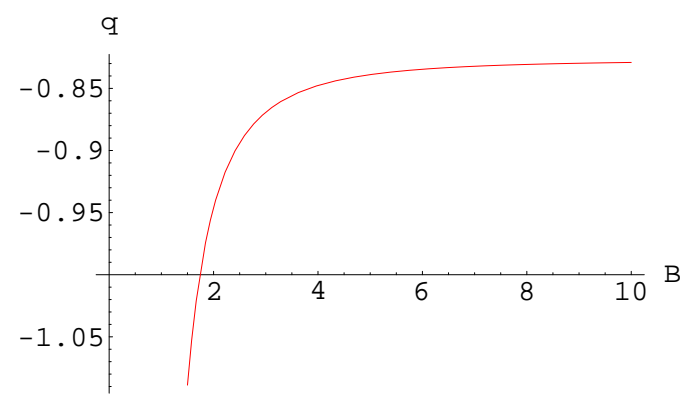

Figure 6: Variation of $q$ against $B$ for $\omega_{m}=0.01, \rho_{m_{0}}=1, H_{1}=1, c=2$, and $\alpha=0.1$.

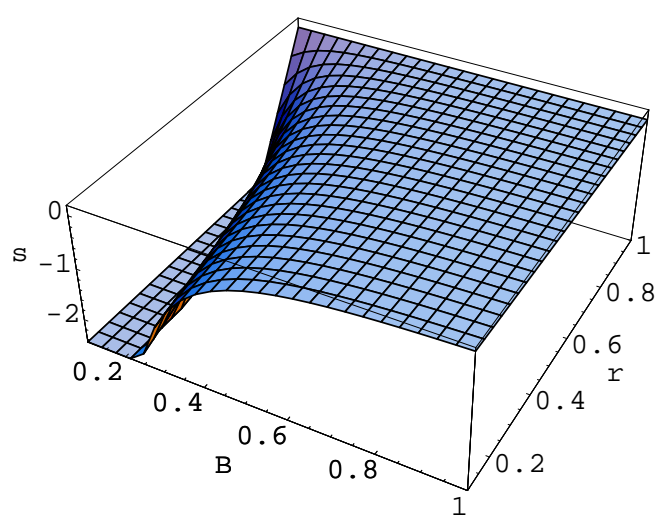

Figure 7: Variation of $s$ against $r$ and $B$ for $\omega_{m}=0.01, \rho_{m_{0}}=1, H_{1}=$ $1, c=2$ and $\alpha=0.1$. 
We plot graphs of $\omega_{h}$ and $\omega$ against the scale factor $B$ in Figures 4 and $\mathbf{5}$, respectively for different values of $\alpha$, which represent the evolution of the universe. Also, the graphs of $q$ against $B$ as well as $s$ against $r$ and scale factor $B$ are shown in Figures $\mathbf{6}$ and $\mathbf{7}$, respectively. The deceleration parameter also generates negative sign and represents expansion of the universe. Figure 7 indicates that $s$ increases from negative to positive values as $r$ decreases and $B$ increases. We conclude that the non-interacting case yields DE.

\subsection{Interacting Case}

Now we assume that the universe is a mixture of GHDE and DM interacting with each other. The conservation equation (6) takes the form

$$
\begin{aligned}
\dot{\rho}_{m}+(m+2) H_{2}\left(\rho_{m}+p_{m}\right) & =-(m+2) \delta H_{2} \rho_{m}, \\
\dot{\rho}_{h}+(m+2) H_{2}\left(\rho_{h}+p_{h}\right) & =(m+2) \delta H_{2} \rho_{m},
\end{aligned}
$$

where $(m+2) \delta H_{2} \rho_{m}$ is interaction, $\delta$ is known as interaction parameter. Solving Eq.(28), we obtain matter density as

$$
\rho_{m}=\rho_{m_{1}} B^{-(m+2)\left(1+\omega_{m}+\delta\right)},
$$

where $\rho_{m_{1}}$ is the constant of integration. Equations (13), (20) and (30) yield the following solution

$$
H_{2}^{2}=\frac{\rho_{m_{1}} B^{-(m+2)\left(1+\omega_{m}+\delta\right)}}{-6 \alpha c^{2}(m+2)^{2}\left[\left[\frac{\mu}{(m+2)}+1\right]+\left(\omega_{m}+\delta\right)\right]}+\left(H_{2}^{\prime}\right)^{2} B^{\mu},
$$

where $H_{2}^{\prime}$ is another integration constant. The corresponding value of energy density and pressure can be obtained by combining Eqs.(20) and (29)-(31) as follows

$$
\begin{aligned}
& \rho_{h}=\gamma_{0} \rho_{m_{1}} B^{-(m+2)\left(1+\omega_{m}+\delta\right)}+(1+2 m)\left(H_{2}^{\prime}\right)^{2} B^{\mu}, \\
& p_{h}=\gamma_{0} \rho_{m_{1}} \omega_{m} B^{-(m+2)\left(1+\omega_{m}+\delta\right)}-(1+2 m)\left[\frac{\mu}{(m+2)}+1\right]\left(H_{2}^{\prime}\right)^{2} B^{\mu},
\end{aligned}
$$

where

$$
\gamma_{0}=\frac{(1+2 m)+6 \alpha c^{2}(m+2)^{2}\left[\left[\frac{\mu}{(m+2)}+1\right]+\left(\omega_{m}+\delta\right)\right]}{-6 \alpha c^{2}(m+2)^{2}\left[\left[\frac{\mu}{(m+2)}+1\right]+\left(\omega_{m}+\delta\right)\right]}
$$




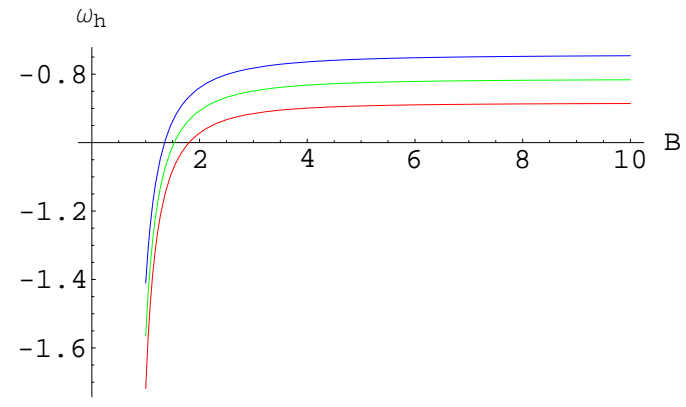

Figure 8: Variation of $\omega_{h}$ against $B$ for $\omega_{m}=0.01, \rho_{m_{1}}=1, H_{2}^{\prime}=1, c=$ $2, \delta=0.01$, and $\alpha=0.1,0.12,0.15$.

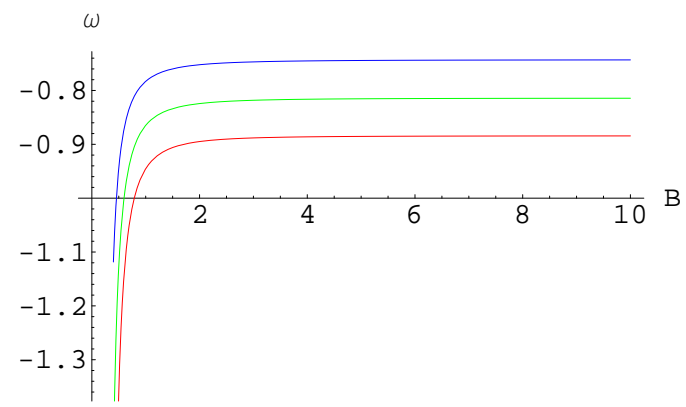

Figure 9: Variation of $\omega$ against $B$ for $\omega_{m}=0.01, \rho_{m_{1}}=1, H_{2}^{\prime}=1, c=$ $2, \delta=0.01$, and $\alpha=0.1,0.12,0.15$.

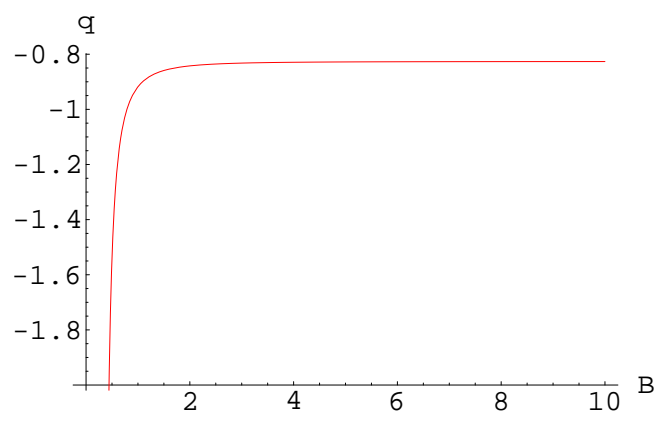

Figure 10: Variation of $q$ against $B$ for $\omega_{m}=0.01, \rho_{m_{1}}=1, H_{2}^{\prime}=1, c=$ $2, \delta=0.01, \alpha=0.1$. 


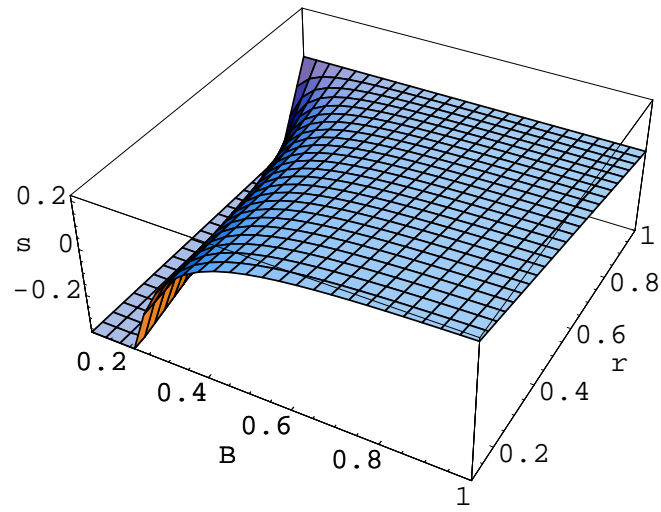

Figure 11: Variation of $s$ against $r$ and $B$ for $\omega_{m}=0.01, \rho_{m_{1}}=1, H_{2}^{\prime}=$ $1, c=2, \delta=0.01, \alpha=0.1$.

The EoS for GHDE can be expressed as

$$
\omega_{h}=\frac{\gamma_{0} \rho_{m_{1}}\left(\omega_{m}+\delta\right) B^{-(m+2)\left(1+\omega_{m}+\delta\right)}-(1+2 m)\left[\frac{\mu}{(m+2)}+1\right]\left(H_{2}^{\prime}\right)^{2} B^{\mu}}{\gamma_{0} \rho_{m_{1}} B^{-(m+2)\left(1+\omega_{m}+\delta\right)}+(1+2 m)\left(H_{2}^{\prime}\right)^{2} B^{\mu}},
$$

and for interacting two fluid system, it takes the form

$$
\begin{aligned}
\omega & =\frac{1}{\left(\gamma_{0}+1\right) \rho_{m_{1}} B^{-(m+2)\left(1+\omega_{m}+\delta\right)}+(1+2 m)\left(H_{2}^{\prime}\right)^{2} B^{\mu}} \\
& \times\left(\gamma_{0}+1\right) \rho_{m_{1}}\left(\omega_{m}+\delta\right) B^{-(m+2)\left(1+\omega_{m}+\delta\right)}-(1+2 m)\left(H_{2}^{\prime}\right)^{2} B^{\mu} \\
& \times\left[\frac{\mu}{(m+2)}+1\right] .
\end{aligned}
$$

The graphs (8)-(11) indicate that the interacting case also provides the accelerated universe as all the above mentioned parameters are negative and generates DE.

\section{GHDE Model with Generalized Chaplygin Gas}

The GCG is a route of investigation in which DM and DE are described within one component model. This model predicts small scale instabilities 
and oscillations at the perturbation level. The GCG behaves like dust matter at early times and behaves like a cosmological constant at late times. In [25, a correspondence between HDE and CG has been established and showed that HDE could be described by a scalar field in a certain way. The GCG has interesting features like it has negative pressure and explains the transition of our universe from decelerating to accelerating phase. Keeping this motivation in mind, we consider generalized HDE and GCG for interacting and noninteracting scenarios.

\subsection{Non-Interacting Case}

First we take the universe which is filled with GHDE and GCG in the noninteracting case. The GCG is a perfect fluid given by [26]

$$
p_{c}=\frac{-A}{\rho_{c}^{\gamma}}, \quad A>0, \quad 0 \leq \gamma \leq 1
$$

Equation (5) can be written in the following form

$$
H_{2}^{2}=\frac{1}{1+2 m}\left(\rho_{h}+\rho_{c}\right)
$$

In this case, the conservation equation (6) for GHDE and GCG become

$$
\dot{\rho}_{c}+(m+2)\left(\rho_{c}+p_{c}\right) H_{2}=0, \quad \dot{\rho}_{h}+(m+2)\left(\rho_{h}+p_{h}\right) H_{2}=0 .
$$

Solving the first of Eq.(38), we get energy density of GCG

$$
\rho_{c}=\left[A+\rho_{c_{0}} B^{-(m+2)(1+\gamma)}\right]^{\frac{1}{1+\gamma}},
$$

where $\rho_{c_{0}}$ is an integration constant. Equation (36) leads to pressure of GCG

$$
\begin{aligned}
p_{c} & =\rho_{c_{0}} B^{-(m+2)(1+\gamma)}\left[A+\rho_{c_{0}} B^{-(m+2)(1+\gamma)}\right]^{\frac{-\gamma}{1+\gamma}} \\
& -\left[A+\rho_{c_{0}} B^{-(m+2)(1+\gamma)}\right]^{\frac{1}{1+\gamma}} .
\end{aligned}
$$

Inserting Eqs.(39) and (40) in (37), we obtain

$$
H_{2}^{2}=\frac{1}{6 \alpha c^{2}(m+2)} e^{\mu x} \int\left[A+\rho_{c_{0}} e^{-(m+2)(1+\gamma) x}\right]^{\frac{1}{1+\gamma}} e^{-\mu x} d x+H_{c_{0}}^{2} B^{\mu},
$$


where $x=\ln B$ and $H_{c_{0}}$ is another integration constant. The corresponding GHDE density is obtained from Eqs.(37), (38) and (41) as

$$
\rho_{h}=\frac{(1+2 m)}{6 \alpha c^{2}(m+2)} e^{\mu x} I(x)-\left[A+\rho_{c_{0}} B^{-(m+2)(1+\gamma)}\right]^{\frac{1}{1+\gamma}}+(1+2 m) H_{c_{0}}^{2} B^{\mu},
$$

where

$$
I(x)=\int\left[A+\rho_{c_{0}} e^{-(m+2)(1+\gamma) x}\right]^{\frac{1}{1+\gamma}} e^{-\mu x} d x .
$$

The conservation equation of GHDE yields

$$
p_{h}=-\rho_{h}-\frac{1}{(m+2)} \frac{\partial \rho_{h}}{\partial x} .
$$

Equations (42) and (43) lead to

$$
p_{h}=\frac{(1+2 m)}{6 \alpha c^{2}(m+2)} e^{\mu x} I(x)-\left[A+\rho_{c_{0}} B^{-(m+2)(1+\gamma)}\right]^{\frac{1}{1+\gamma}}+(1+2 m) H_{c_{0}}^{2} B^{\mu} .
$$

The EoS for GHDE is

$$
\begin{aligned}
\omega_{h} & =(1+2 m)\left[\frac{I(x)}{6 \alpha c^{2}(m+2)}+H_{c_{0}}^{2}\right] y(x)-\left[A+\rho_{c_{0}} B^{-(m+2)(1+\gamma)}\right]^{-\frac{1}{1+\gamma}} \\
& \times\left[-\frac{(1+2 m)}{6 \alpha c^{2}(m+2)^{2}}\left[A+\rho_{c_{0}} B^{-(m+2)(1+\gamma)}\right]^{\frac{1}{1+\gamma}}-(1+2 m)\left[\frac{\mu}{(m+2)}+1\right]\right. \\
& \left.\times\left[\frac{I(x)}{6 \alpha c^{2}(m+2)}+H_{c_{0}}^{2}\right] y(x)+A\left[A+\rho_{c_{0}} B^{-(m+2)(1+\gamma)}\right] \frac{-\gamma}{1+\gamma}\right]
\end{aligned}
$$

where $y(x)=e^{\mu x}$. The EoS for combined fluid is

$$
\begin{aligned}
\omega & =\frac{1}{(1+2 m)\left[\frac{I(x)}{6 \alpha c^{2}(m+2)}+H_{c_{0}}^{2}\right] y(x)} \times\left[-(1+2 m)\left[\frac{\mu}{(m+2)}+1\right]\right. \\
& \times\left[\frac{I(x)}{6 \alpha c^{2}(m+2)}+H_{c_{0}}^{2}\right] y(x)-\frac{(1+2 m)}{6 \alpha c^{2}(m+2)^{2}}\left[A+\rho_{c_{0}} B^{-(m+2)(1+\gamma)}\right]^{\frac{1}{1+\gamma}} \\
& \left.-(1+2 m)\left[\frac{\mu}{(m+2)}+1\right] H_{c_{0}}^{2} B^{\mu}\right] .
\end{aligned}
$$

The behavior of $\omega_{h}$ for GHDE is shown in Figure 12 for appropriate values which represents the expanding universe by keeping negative sign. Figure 13 shows the variation of $\omega$ against $B$ indicating $\omega$ as negative. The graphs of $q$ against $B$ and $s$ against $r$ and $B$ are plotted in Figures 14 and 15, respectively. The deceleration parameter also possesses negative sign and generates DE and $s$ has increasing behavior for decreasing $r$ and increasing $B$. 


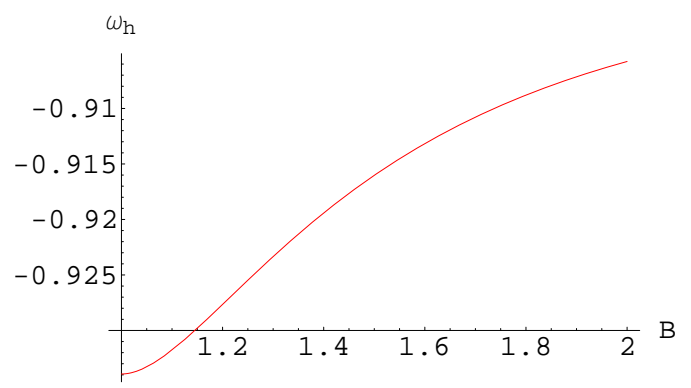

Figure 12: Variation of $\omega_{h}$ against $B$ for $\rho_{c_{0}}=1, H_{c_{0}}=1, c=2, \gamma=$ $0.1, \alpha=0.1, A=1$.

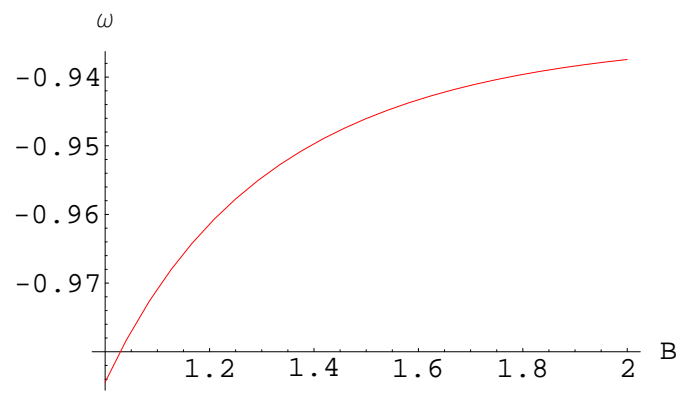

Figure 13: Variation of $\omega$ against $B$ for $\rho_{c_{0}}=1, H_{c_{0}}=1, c=2, \gamma=$ $0.1, \alpha=0.1, A=1$.

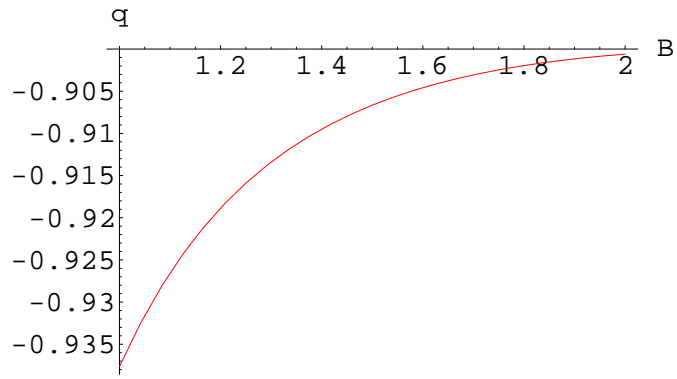

Figure 14: Variation of $q$ against $B$ for $\rho_{c_{0}}=1, H_{c_{0}}=1, c=2, \gamma=0.1, \alpha=$ $0.1, A=1$. 


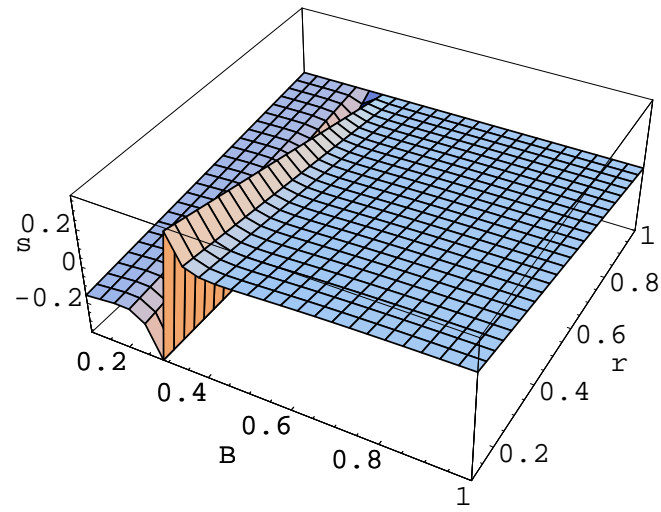

Figure 15: Variation of $s$ against $r$ and $B$ for $\rho_{c_{0}}=1, H_{c_{0}}=1, c=2, \gamma=$ $0.1, \alpha=0.1, A=1$.

\subsection{Interacting Case}

Now we assume that the universe is a combination of GHDE and GCG interacting each other. The equations of conservation for interacting GHDE and GCG become

$$
\begin{aligned}
\dot{\rho}_{c}+(m+2) H_{2}\left(\rho_{c}+p_{c}\right) & =-(m+2) \delta H_{2} \rho_{c} \\
\dot{\rho}_{h}+(m+2) H_{2}\left(\rho_{h}+p_{h}\right) & =(m+2) \delta H_{2} \rho_{c} .
\end{aligned}
$$

Using EoS of GCG in Eq.(48) and after solving, we obtain

$$
\rho_{c}=\left[\frac{A}{1+\delta}+\rho_{c_{1}} B^{-(m+2)(1+\gamma)(1+\delta)}\right]^{\frac{1}{1+\gamma}},
$$

where $\rho_{c 1}$ is the constant of integration. From Eq.(36), we obtain

$$
p_{c}=-A\left[\frac{A}{1+\delta}+\rho_{c_{1}} B^{-(m+2)(1+\gamma)(1+\delta)}\right]^{\frac{-\gamma}{1+\gamma}} .
$$

Inserting $\rho_{c}$ in Eq.(37), we finally obtain

$$
\begin{aligned}
H_{2}^{2} & =\frac{1}{6 \alpha c^{2}(m+2)} e^{\mu x} \int\left[\frac{A}{1+\delta}+\rho_{c_{1}} e^{-(m+2)(1+\gamma)(1+\delta) x}\right]^{\frac{1}{1+\gamma}} e^{-\mu x} d x \\
& +H_{c_{1}}^{2} B^{\mu}
\end{aligned}
$$




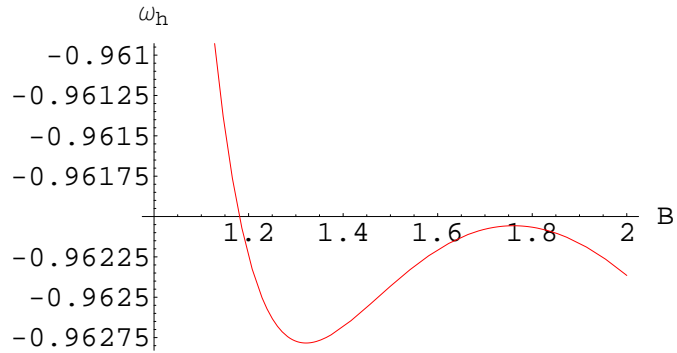

Figure 16: Variation of $\omega_{h}$ against $B$ for $\rho_{c_{1}}=1, H_{c_{1}}=1, c=2, \gamma=$ $0.1, \alpha=0.1, A=1, \delta=0.01$.

where $H_{c_{1}}$ is another constant of integration. Thus we have

$$
\begin{aligned}
\rho_{h} & =\frac{(1+2 m)}{6 \alpha c^{2}(m+2)} e^{\mu x} J(x)-\left[\frac{A}{1+\delta}+\rho_{c_{1}} B^{-(m+2)(1+\gamma)(1+\delta)}\right]^{\frac{1}{1+\gamma}} \\
& +(1+2 m) H_{c_{1}}^{2} B^{\mu}
\end{aligned}
$$

where

$$
J(x)=\int\left[\frac{A}{1+\delta}+\rho_{c_{1}} e^{-(m+2)(1+\gamma)(1+\delta) x}\right]^{\frac{1}{1+\gamma}} e^{-\mu x} d x .
$$

The energy conservation equation gives

$$
p_{h}=\delta \rho_{c}-\rho_{h}-\frac{1}{(m+2)} \frac{\partial \rho_{h}}{\partial x}
$$

Using Eq.(49) and (52), it follows that

$$
\begin{aligned}
p_{h} & =-(1+2 m)\left[\frac{\mu}{(m+2)}+1\right]\left[\frac{J(x)}{6 \alpha c^{2}(m+2)}-H_{c_{1}}^{2}\right] y(x) \\
& +\left[\delta-\frac{(1+2 m)}{6 \alpha c^{2}(m+2)}\right]\left[\frac{A}{1+\delta}+\rho_{c 1} B^{-(m+2)(1+\gamma)(1+\delta)}\right]^{\frac{1}{1+\gamma}} \\
& +\left[\frac{A}{1+\delta}-\delta \rho_{c_{1}} B^{-(m+2)(1+\gamma)(1+\delta)}\right] \\
& \times\left[\frac{A}{1+\delta}+\rho_{c 1} B^{-(m+2)(1+\gamma)(1+\delta)}\right]^{\frac{-\gamma}{1+\gamma}}
\end{aligned}
$$

where $y(x)=e^{\mu x}$. 


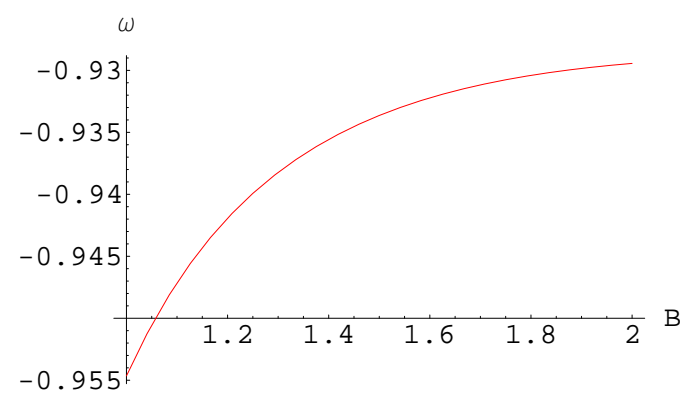

Figure 17: Variation of $\omega$ against $B$ for $\rho_{c_{1}}=1, H_{c_{1}}=1, c=2, \gamma=$ $0.1, \alpha=0.1, A=1, \delta=0.01$.

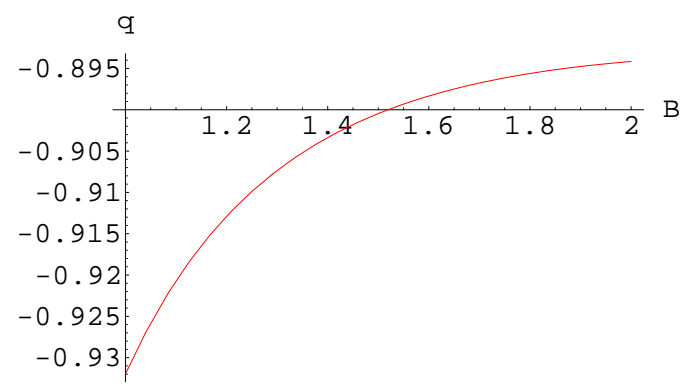

Figure 18: Variation of $q$ against $B$ for $\rho_{c_{1}}=1, H_{c 1}=1, c=2, \gamma=0.1, \alpha=$ $0.1, A=1, \delta=0.01$.

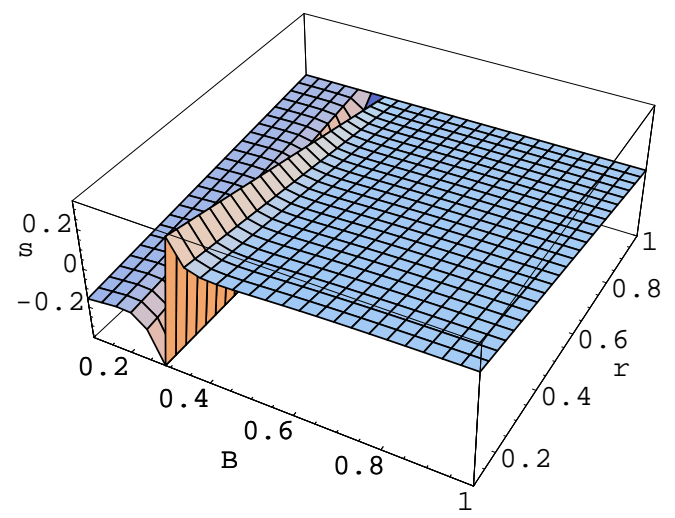

Figure 19: Variation of $s$ against $r$ and $B$ for $\rho_{c_{1}}=1, H_{c 1}=1, c=2, \gamma=$ $0.1, \alpha=0.1, A=1, \delta=0.01$. 
The EoS for GHDE $\omega_{h}$ is expressed as

$$
\begin{aligned}
\omega_{h} & =\frac{1}{(1+2 m)\left[\frac{\mu}{(m+2)}+1\right]\left[\frac{J(x)}{6 \alpha c^{2}(m+2)}-H_{c_{1}}^{2}\right] y(x)-\left[\frac{A}{1+\delta}+\rho_{c_{1}} B^{-(m+2)(1+\gamma)(1+\delta)}\right]^{\frac{1}{1+\gamma}}} \\
& \times-(1+2 m)\left[\frac{\mu}{(m+2)}+1\right]\left[\frac{J(x)}{6 \alpha c^{2}(m+2)}-H_{c_{1}}^{2}\right] y(x) \\
& +\left[\delta-\frac{(1+2 m)}{6 \alpha c^{2}(m+2)}\right]\left[\frac{A}{1+\delta}+\rho_{c_{1}} B^{-(m+2)(1+\gamma)(1+\delta)}\right]^{\frac{1}{1+\gamma}} \\
& +\left[\frac{B}{1+\delta}-\delta \rho_{c_{1}} B^{-(m+2)(1+\gamma)(1+\delta)}\right]\left[\frac{B}{1+\delta}+\rho_{c_{1}} B^{-(m+2)(1+\gamma)(1+\delta)}\right]^{\frac{-\gamma}{1+\gamma}} .
\end{aligned}
$$

The EoS for interacting GHDE and GCG has the form

$$
\begin{aligned}
\omega & =\frac{1}{(1+2 m)\left[\frac{\mu}{(m+2)}+1\right]\left[\frac{J(x)}{6 \alpha c^{2}(m+2)}-H_{c_{1}}^{2}\right] y(x)-\left[\frac{A}{1+\delta}+\rho_{c_{1}} B^{-(m+2)(1+\gamma)(1+\delta)}\right]^{\frac{1}{1+\gamma}}} \\
& \times-(1+2 m)\left[\frac{\mu}{(m+2)}+1\right]\left[\frac{J(x)}{6 \alpha c^{2}(m+2)}+H_{c_{1}}^{2}\right] y(x) \\
& -\frac{(1+2 m)}{6 \alpha c^{2}(m+2)^{2}}\left[\frac{A}{1+\delta}+\rho_{c_{1}} B^{-(m+2)(1+\gamma)(1+\delta)}\right]^{\frac{1}{1+\gamma}}
\end{aligned}
$$

All the parameters are shown in Figures (16)-(19). These plots indicate that this model also generates DE for interacting case.

\section{Concluding Remarks}

The main purpose of this paper is to check the role of EoS, deceleration, and statefinder parameters in the accelerated universe. To this end, we have considered the LRS BI universe model with two models of DE, i.e., GHDE/GRDE. We have assumed different cases like combined DM and DE fluid, GHDE/GRDE without and with DM (both interacting and noninteracting) as well as GHDE/GRDE without and with GCG (both interacting and non-interacting) to investigate evolution of the universe. In all these cases, the deceleration, EoS and statefinder parameters are calculated which help to explore the accelerating universe.

We have displayed all the parameters graphically by using appropriate values of the constants to understand their behavior. The EoS and deceleration parameters contain negative sign for both interacting and non-interacting 
scenarios which support the acceleration of the universe. The graphical behavior of statefinder parameters shows that $s$ always increases as $r$ decreases and $B$ increases for specified values. The general nature of the parameters indicate the accelerating behavior of the universe. We would like to mention here that the nature of GRDE model can be obtained by replacing $\alpha$ with $1-\beta$ in GHDE model. We have also calculated the values of $\alpha$ and $\beta$ for accelerating universe. It has been found that our universe will be accelerating if $\alpha<\frac{(m+2)^{2} c^{2}-3(1+2 m)}{\left(7 m^{2}-8 m+10\right) c^{2}}$ for GHDE and $\beta>\frac{\left(6 m^{2}-12 m+6\right) c^{2}+3(1+2 m)}{\left(7 m^{2}-8 m+10\right) c^{2}}$ for GRDE. We conclude that statefinder parameters have an extra contribution of EoS parameter $\omega$ as compared to FRW. The statefinder diagnostic pair also represents the $\Lambda C D M$ model.

Finally, we would like to mention here that our work supports the results of a recent paper [22] for FRW model, where all the parameters represent $\mathrm{DE}$ and evolution of the universe.

\section{References}

[1] Perlmutter, S. et al.: Astron. Soc. 29(1997)1351; Nature 391(1998)51; Astrophys. J. 517(1999)565; Riess, A.G. et al.: Astron. J. 116(1998)1009.

[2] Spergel, D.N. et al.: Astrophys. J. Suppl. 170(2007)377.

[3] Tegmark, M. et al.: Phys. Rev. D69(2004)03501.

[4] Bennett, C.L. et al.: Astrophys. J. Suppl. 148(2003)1; Peebles, P.J.E. and Ratra, B.: Rev. Mod. Phys. 75(2003)559.

[5] Ratra, B. and Peebles, P.J.E.: Phys. Rev. D37(1998)3406; Padmanabhan, T.: Gen. Relativ. Gravit. 40(2008)529.

[6] Chiba, T., Okabe, T. and Yamaguchi, M.: Phys. Rev. D62(2000)023511.

[7] Sen, A.: JHEP 48(2002)204; Padmanabhan, T.: Phys. Rev. D66(2002)021301.

[8] Caldwell, R.R.: Phys. Lett. B545(2002)23; Nojiri, S. and Odintsov, S.D.: Phys. Lett. B562(2003)147; ibid. B565(2003)1. 
[9] Bento, M.C., Bertolami, O. and Sen, A.A.: Phys. Rev. D66(2002)043507; Liu, D.J. and Li, X.Z.: Chin. Phys. Lett. $22(2005) 1600$.

[10] Susskind, L.: J. Math. Phys. 36(1995)6377.

[11] Cohen, A., Kaplan, D. and Nelson, A.: Phys. Rev. Lett. 82(1999)4971.

[12] Hsu, S.D.H.: Phys. Lett. B594(2004)13; Li, M.: Phys. Lett. B603(2004)1.

[13] Zhang, X.: Int. J. Mod. Phys. D14(2005)1597.

[14] Li, H., Guo, Z.K. and Zhang, Y.Z.: Int. J. Mod. Phys. D15(2006)869; Almeida, J.P.B. and Pereira, J.G.: Phys. Lett. B636(2006)75; Gong, Y.: Phys. Rev. D70(2004)064029.

[15] Gao, C., Wu, F., Chen, X. and Shen, Y. G.: Phys. Rev. D79(2009)043511.

[16] Chattopadhyay, S.: Europ. Phys. J. Plus 127(2012)16.

[17] Feng, C.J. and Li, X.Z.: Phys. Lett. B680(2009)355.

[18] Cai, R.G., Hu, B. and Zhang, Y.: Commun. Theor. Phys. 51(2009)954.

[19] Kim, K.Y., Lee, H.W., Myung, Y.S.: Gen. Relativ. Gravit. $\mathbf{4 3}(2011) 1095$.

[20] Sadjadi, H.M. and Vadood, N.: JCAP 0808(2008)036; Saridakis, E.N.: Phys. Lett. B661(2008)335; Granda, I.N. and Oliveros, A.: Phys. Lett. B669(2008)275; Feng, C.J. Phys. Lett. B672(2009)94; Jamil, M., Farooq, M.U. and Rashid, M.A.: Eur. Phys. J. C61(2009)471.

[21] Xu, L., Lu, J. and Li, W.: Eur. Phys. J. C64(2009)89.

[22] Khatua, P.B. and Debnath. U.: arXiv:1106.5689.

[23] Sharif, M. and Zubair, M.: Astrophys. Space Sci. 330(2010)399; Sharif, M. and Waheed, S.: Eur. Phys. J. C72(2012)1876.

[24] Sahni, V., Saini, T., Starobinsky, A.A., and Alam, U.: JETP Lett. $\mathbf{7 7}(2003) 201$. 
[25] Setare, M. R.: Phys. Lett. B648(2007)329.

[26] Gorini, V., Kamenshchik, A. and Moschella, U.: Phys. Rev. D67(2003)063509; Alam, U., Sahni, V., Saini, T.D., and Starobinsky, A.A.: Mon. Not. Roy. Astron. Soc. 344(2003)1057. 